\title{
Caractéristiques biologiques de la crevette d'eau douce Atyaephyra desmaresti Millet dans la Meuse
}

\author{
M. Meurisse-Génin ${ }^{1}$ \\ A. Reydams-Detollenaere ${ }^{1}$ \\ O. Donatitil \\ J.C. Michal
}

Mots-clés : Croissance, Cycle de vie, Reproduction, Sex-ratio, Régime alimentaire.

A partir d'échantillons de crevettes capturées par pêche à l'électricité de 1977 à 1980, les principales caractéristiques de la population d'Atyaephyra desmaresti de la Meuse ont été décrites et analysées. Ainsi, l'abondance de ce crustacé diminue au cours des années étudiées; le sex-ratio femelles/mâles moyen est de 1,6. C'est une espèce univoltine, le recrutement a lieu en été. Des données concernant la durée de vie, les croissances en longueur (Longueurs totales maximales des femelles et des máles : 36 et $29 \mathrm{~mm}$ ), et en poids (Poids maxima atteints par les femelles et les mâles: 250 et $137 \mathrm{mg}$ ) unt été analysées. La période de reproduction s'étend sur $\mathbf{4}$ mois : de mai à août. La taille minimale des femelles à maturite est de $22 \mathrm{~mm}$. La relation entre la fécondité et la taille de la crevette est de type linéaire. Enfin, le régime alimentaire dé cette espèce est essentiellement détritivore.

Biological characteristics of a freshwater shrimp Atvephyra desmaresti Millet in the River Meuse

Keywords : Growth, Life cycle, Reproduction, Sex ratio, Diet.

From samples of shrimps captured by electro-fishing in 1977 to 1980, the principal characteristics of the population of Atvaephyra desmaresti in the Meuse have been described and analysed. Thus, the abundance of the shrimp decreased during the course of the years studies; the mean sex ratio of females to males was 1.6. It is a univoltine species with recruitment taking place in summer. Data are provided for longevity, growth in length (total maximum length for females and males is $36 \mathrm{~mm}$ and $29 \mathrm{~mm}$ ) and weight (maximum weight of females and males is $250 \mathrm{mg}$ and $137 \mathrm{mg}$ ). The reproductive period lasts four months from May to August. The minimum size of mature females is $22 \mathrm{~mm}$. The relationship between fecundity and shrimp size is linear. Finally, the food of this species is chiefly detritus.

\section{1. - Introduction}

Atyephyra desmaresti, crevette d'eau douce d'origine méditerranéenne, a envahi le nord de l'Europe par la voie des canaux. Elle fut signalée pour la pre. mière fois en Belgique par Pelseneer en 1886 (in Redeke 1936), et actuellement, on la rencontre dans de nombreux canaux et rivières canalisées (Lestage 1931, Adam \& Leloup 1940). D'après Stephen (1939, in Packa Tchissambou 1979), la dispersion de l'espèce s'effectue, essentiellement, à partir des stades larvaires planctoniques.

I. Unité d'Ecologie des Eaux Douces (UNECED), Facultés Universitaires N.-D. de la Paix, rue de Bruxelles, 61 B-5000 Namur. Belgique.
L'importance de ce crustacé dans la chaîne trophique de l'écosystème fluviatile, en tant que maillon intermédiaire entre les végétaux et les poissons, nous a incités à étudier différents paramètrés de la biologie de cette espèce. Ainsi, le cycle vital, la croissance en longueur et en poids de cette crevette de la Meuse namuroise ont été étudiés depuis fin 1977 jusque fin 1980 . Les phénomènes liés à la reproduction (période de reproduction et fécondité) ont été également examinés et une approche du régime alimentaire a été réalisée dans le but de mieux connaître le rôle de l'espèce dans l'écosystème mosan.

Dans le cadre d'une étude d'impact de la centrale nucléaire de Tihange sur la faune aquatique de la Meuse (contrat C.E.E. - C.E.N. - I.H.E. BIO-B - 


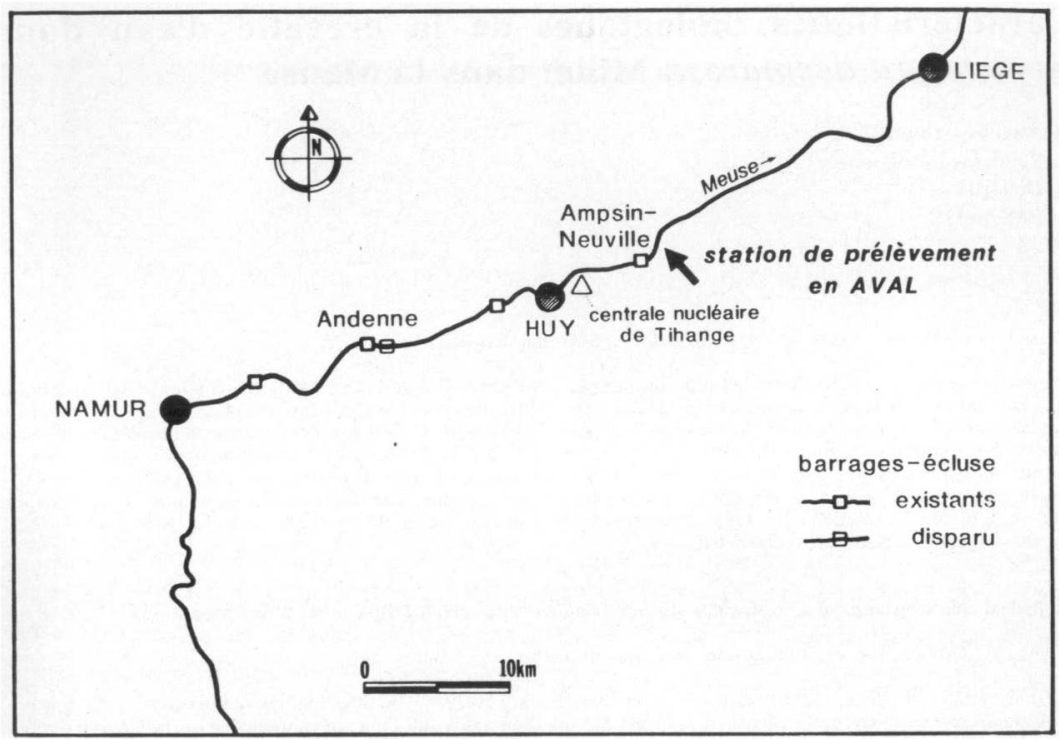

Fig. 1. Localisation de la station de prélèvement.

$330-81 \mathrm{~B}$ ), une recherche des caractéristiques biologiques de la crevette Atyephyra desmaresti Mil. let a été ent reprise dans la Meuse. En raison des problèmes liés à l'échantillonnage, ces différents paramètres ont été uniquement étudiés sur les crevettes capturées en aval.

\section{2. - Caractéristiques}

La meuse coule dans un bassin versant de 41400 $\mathrm{km}^{2}$ dont $17300 \mathrm{~km}^{2}$ en Belgique $(41,8 \%), 12300$ $\mathrm{km}^{2}$ en France $(29,7 \%), 7300 \mathrm{~km}^{2}$ aux Pays-Bas et $4500 \mathrm{~km}^{2}$ en République Fédérale d'Allemagne. D'une longueur toale de 925 km (492 km en France, $194 \mathrm{~km}$ en Belgique, $239 \mathrm{~km}$ aux Pays-Bas), ce fleuve prend sa source en France sur le plateau de Langres à une altitude de $402 \mathrm{~m}$. Il pénètre en belgique à
Hastière, coule du Sud au Nord jusque Namur, puis prend une direction N-E jusqu'aux Pays-Bas.

En Belgique, à mi-distance entre Namur et Liège, juste en aval de Huy, fonctionne depuis 1975 la centrale nucléaire de Tihange (Tihange 1:872 MWe).

C'est en aval de celle-ci à Amay-Ombret, que se situe la station d'échantillonnage (fig. 1) de Atyephyra desmaresti, petit crustacé de la famille des Atyidés et de l'ordre des Décapodes.

Le régime thermique de la Meuse, après réchauffement par la centrale varie entre $2^{\circ} \mathrm{C}$ et $27^{\circ} \mathrm{C}$ au cours des années 1977-1980.

Les températures minimales sont mesurées pendant les mois de janvier et février. Puis, suite à un réchauffement naturel de l'eau, la température observée durant les mois de mars et avril avoisine 
Tableau I. Caractéristiques physico-chimiques principales des eaux de la Meuse à Tihange, en aval de la centrale, pendant la période 1977-1980 (Declercq-Versele H., Hirchmann R. \& al. 1982).

\begin{tabular}{|c|c|c|c|}
\hline Paramètres & Minimurn & Maximum & Moyenne \\
\hline pH & 7,4 & 8,4 & 7,9 \\
\hline Oxygène $(\mathrm{mg} / \mathrm{l})$ & 6,9 & 13,7 & 9,6 \\
\hline Oxygène ( $\%$ sat.) & 72,6 & 131,0 & 93,3 \\
\hline Conductivite $(\mu \mathrm{S} / \mathrm{cm})$ & 339 & 950,0 & 577,4 \\
\hline Alcalinité $\left(\mathrm{mg} / \mathrm{l} \mathrm{CaCO}_{3}\right)$ & 124,0 & 218,0 & 166,1 \\
\hline T.O.C. (mg/l c) & 2,1 & 27,0 & 6,1 \\
\hline Chlorures (mg/t) & 24,0 & 138,0 & 57,5 \\
\hline Nitrates (mg/l N) & 1,6 & 4,2 & 3,0 \\
\hline Phosphates (ppb P) & 37,0 & 455,0 & 217,3 \\
\hline Sulfates $(\mathrm{mg} / \mathrm{l})$ & 36,0 & 71,0 & 53,3 \\
\hline Calcium (mg/l) & 62,0 & 97,4 & 78,5 \\
\hline Magnésium (mg/l) & 5.6 & 9,8 & 7,3 \\
\hline Sodium (mgl) & 10,3 & 73,9 & 28,5 \\
\hline Ammoniaque (ppb N) & 130,0 & 897,0 & 364,7 \\
\hline Nitrites (ppb N) & 25,0 & 140,0 & 69,9 \\
\hline
\end{tabular}

les $10^{\circ} \mathrm{C}$. Le réchauffement de l'eau se poursuit ; c'est ainsi qu'en général, lors de la première quinzaine de mai, les $15^{\circ} \mathrm{C}$ sont atteints. Les mois de juin, juillet en août sont caractérisés par des températures souvent supérieures à $20^{\circ} \mathrm{C}\left(\max .: 27^{\circ} \mathrm{C}\right)$. Ensuite, après une diminution progressive au cours des mois de septembre et octobre (température comprise entre 15 et $20^{\circ} \mathrm{C}$ ), la température de l'eau tombe sous les $10^{\circ} \mathrm{C}$ en novembre et décembre.

Le réchauffement de l'eau de la Meuse par les rejets de la centrale nucléaire de Tihange est de l'ordre de 2 à $3^{\circ} \mathrm{C}$ (Declercq-Versele \& Kirchmann 1982). Toutefois, des valeurs de 4 à $5^{\circ} \mathrm{C}$ peuvent être atteintes en automne, au cours du debit d'étiage. Les principales données physico-chimiques des eaux de la Meuse sur ce secteur sont reprises dans le tableau I. Le débit moyen annuel du fleuve pendant ces 4 années a été de $220 \mathrm{~m}^{3} / \mathrm{sec}$.

La zone de prélèvement s'étend sur ụne longueur de $2 \mathrm{~km}$. Elle se situe le long des berges à une profondeur variant de $0,10 \mathrm{~m}$ à $1 \mathrm{~m}$, présentant un substrat caillouteux.

\section{3. - Méthodes}

Les crevettes sont capturées par pêche électrique à l'aide d'un groupe électrogène de marque Electropullman $(1,8 \mathrm{kw})$, fournissant un courant continu de
6 A et de $420 \mathrm{~V}$ maximum. La pêche s'effectue sur un canot pneumatique « Astral "(4 pers.), et les crevettes sont prélevées vivantes à l'épuisette (maille : $1 \mathrm{~mm}$ ).

Des prélèvements mensuels ont débuté en octobre 1977 jusque octobre 1980 ; toutefois en période de hautes eaux, les pêches n'ont pas eu lieu. Après capture, les crevettes sont conservées dans une solution de formol à $10 \%$.

Chaque lot de crevettes est ensuite soumis à un sous-échantillonnage.

Celles-ci sont réparties de façon homogène dans un bac blanc quadrillé ( $35 \mathrm{~cm} \times 25 \mathrm{~cm} \times 6 \mathrm{~cm}$ ). Certains carrés sont tirés au hasard afin d'obtenir environ 300 individus. Le rapport des sexes est alors défini sur ces crevettes. Le sexe est déterminé par l'examen sous binoculaire de l'extrémité an térieure du sternite thoracique posterieur. Ce dernier est tronqué en aval chez la femelle, tandis que chez le mâle, il se prolonge antérieurement en stylet (Descouturelle 1971).

Afin d'étudier la croissance d'Atyephyra desmaresti, la taille de chaque individu est déterminée en mesurant sous binoculaire $(G \times 8)$ la distance entre l'extrémité du rostre et du telson après étalement total le long d'une lat te graduée au mm. Ensuite, les organismes sont séchés sur papier filtre et pesćs sur une balance (précision $0,001 \mathrm{~g}$ ). La fécondité des 
Tableau II. Captures par pèche électrique d'Atyephyra desmaresti sur les berges de la Meuse à Amay en 1977, 1978, 1979 et 1980 (temps de pêche : environ 1 heure) $/$ : pas de prèlèvement.

\begin{tabular}{|c|c|c|c|c|c|c|c|c|c|c|c|c|c|}
\hline \multirow[b]{2}{*}{ Année } & \multirow[b]{2}{*}{ Mois } & \multirow[b]{2}{*}{01} & \multirow[b]{2}{*}{02} & \multirow[b]{2}{*}{03} & \multirow[b]{2}{*}{04} & \multicolumn{4}{|c|}{ Nombre d'individus capturés } & \multirow[b]{2}{*}{09} & \multirow[b]{2}{*}{10} & \multirow[b]{2}{*}{11} & \multirow[b]{2}{*}{12} \\
\hline & & & & & & 05 & 06 & 07 & 08 & & & & \\
\hline 1977 & & 1 & 1 & 1 & 1 & 1 & 1 & 1 & j & 1 & 300 & 1 & 300 \\
\hline 1978 & & 1 & 1 & 300 & i & 300 & 300 & 300 & 300 & $i$ & 300 & 300 & 1 \\
\hline 1979 & & 1 & 1 & 1 & 0 & 1 & 295 & 15 & 203 & 300 & 300 & 300 & i \\
\hline 1980 & & 32 & 1 & 170 & 34 & 1 & 350 & $j$ & 0 & 3 & 322 & 1 & 1 \\
\hline
\end{tabular}

femelles est déterminée par le comptage des œufs attachés aux pléopodes.

Quand à l'étude du régime alimentaire, elle est basée sur l'examen du contenu stomacal, placé sous microscope $(G \times 40)$ dans une cellule de Bürker, habituellement utilisée pour compter les cellules sanguines.

L'analyse du contenu stomacal est basée sur 2 méthodes : la méthode d'occurrence qualitative, et la méthode numérique quantitative, employée par Minshall (1966). Sur les 144 carrés de la cellule de Bürker, seuls 20 carrés tirés au hasard ont été analysés.

Le nombre optimal de carrés à examiner a été déterminé en comptant respectivement le nombre d'organismes sur 20, 40,60 et 144 carrés et en comptant les moyennes. Les algues sont identifiées au moyen de clés de détermination de Bourelly (1968, 1970, 1972) et de Prescott (1970). L'identification des diatomées s'est faite après nettoyage de la prépara. tion à l'acide nitrique. les protozoaires ont été identifiés d'après les clés de détermination de Perrier (1971).

\section{4. - Résultats}

\section{1. - Résultats de capture}

Depuis octobre 1977 jusque novembre 1978, des échantillons de crevet te $(\mathbf{n}>300)$ ont été capturés régulièrement sauf en période de crues. A partir de 1979, l'abondance de la caridine a sensiblement diminué sur les berges de la Meuse. En effet, comme l'indique le tableau II, leur capture en 1979 , est plus irrégulière. En 1980, la crevette d'eau douce s'observe encore plus rarement sur les berges; c'est pourquoi l'étude de la croissance s'est uniquement basée sur les organismes capturés en 1977, 78 et 79. Les atyidés capturés en 1980 ont uniquement servi à déterminer le régime alimentaire.

\section{2. - Rapport des sexes}

Sur la base des sous-échantillons examinés aut laboratoire, le rapport des sexes de la population de caridines a été défini. On constate que le rapport $q / \sigma$ est quasi toujours supérieur à 1 (Tableau III). Ce rapport augmente sensiblement au cours des mois de juin et août. Ainsi, à cette période, les femelles sont, dans les échantillons, 2 à 4 fois plus nombreuses que les mâles. De plus, sur l'ensemble des captures, le rapport est de 1,6. La population de caridine semble donc constituée d'une plus grande proportion de femelles.

\section{3. - Croissance en longueur}

Les femelles d'Atyephyra desmaresti étant toujours plus grandes que les mâles (Nouvel 1940, Vorstaman 1955, Bouvier 1925), la croissance a été examinée séparément pour chaque sexe. Les caractères sexuels, décrits précédemment, sont visibles sur les crevetes mesurant au moins 15 à $16 \mathrm{~mm}$.

La figure 2 retrace l'évolution saisonnière des histogrammes de fréquence des tailles de 300 atyidés, en général ( $150 \%$ et $150 \%$ ); nous y avons ajouté les juvéniles de sexe indéterminé, de longueur inférieure ou égale à $16 \mathrm{~mm}$ et dont le nombre a été calculé sur un échantillon de 300 crevettes tirées au 
1977

Décembre $\left(\mathbf{N}=150^{\circ}+1226^{\circ}+2 J\right)$

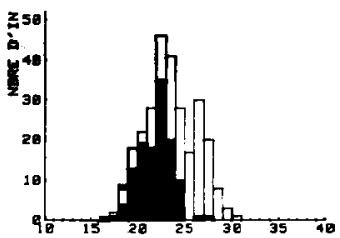

Octobre $(\mathrm{N}=1508+150 \mathrm{~d}+15 \mathrm{~J})$

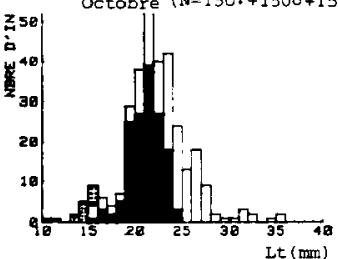

Fenelles 9

Males d

:i Juveñiles $\mathrm{J}$
1978

Novembre $(N=150 q+1508)$

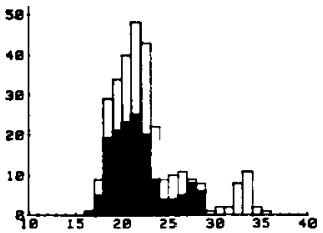

octobre $(N=1509+1508+63 J)$
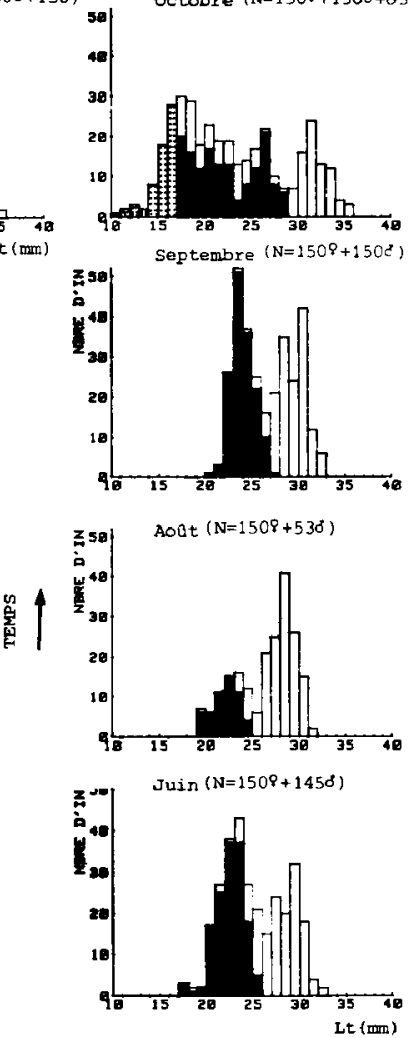

1979

Novembre $(N=1508+150 d+: 2$

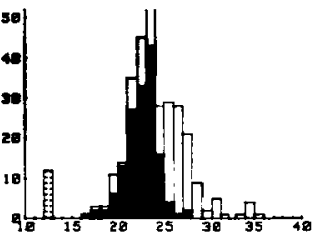

Octobre $(N=150 \%+150 \delta+5 J)$
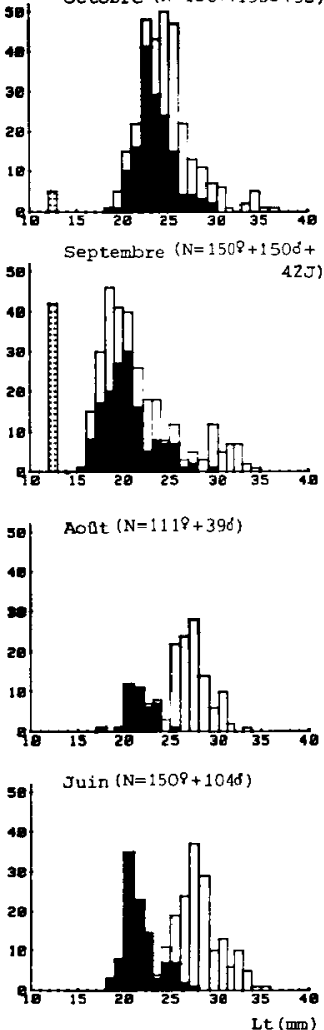

Fig. 2. Evolution des histogrammes de fréquence de taille d'Atyephyra desmaresti au cours de 3 années ( $\sigma$ : mâles, $Q:$ femelles, $J$ : juvéniles). 
Tableau III. Rapport des sexes ( $/$ / $/$ ) des populations d'Atyephyra desmaresti calculé au cours des différents prélèvements. dans la Meuse à Amay.

I. : crevettes juvéniles, de sexe indéterminé

* : à cette date, toutes les crevettes ont été examinées vivantes.

\begin{tabular}{|c|c|c|c|c|c|}
\hline Date & $\mathrm{N}$ & $!$ & q & o & R.S. \\
\hline 12.10 .77 & 314 & 15 & 189 & 110 & 1,7 \\
\hline 16.12 .77 & 295 & 2 & 171 & 122 & 1,4 \\
\hline 09.03 .78 & 300 & - & 147 & 153 & 0,9 \\
\hline 19.05 .78 & 300 & - & 164 & 136 & 1,2 \\
\hline 14.06 .78 & 300 & - & 215 & 85 & 2,5 \\
\hline 02.08 .78 & 300 & - & 247 & 53 & 4,6 \\
\hline 01.09 .78 & 300 & - & 214 & 86 & 2,5 \\
\hline 10.10 .78 & 300 & 63 & 160 & $\pi$ & 2,1 \\
\hline 01.12 .78 & 300 & $\rightarrow$ & 160 & 140. & 1,1 \\
\hline 28.06 .79 & 297 & - & 192 & $105:$ & 1,8 \\
\hline 21.08 .79 & 151 & - & 112 & 839 & 2,9 \\
\hline 26.09 .79 & 823 & 348 & 265 & 230 & 1,1 \\
\hline 25.10 .79 & 301 & 5 & 171 & 125 & 1,4 \\
\hline 26.11 .79 & 309 & 12 & 155 & 142 & 1,1 \\
\hline TOTAL & 4590 & & 2542 & 1603 & 1,6 \\
\hline
\end{tabular}

hasard (Tableau III). Toutefois, en 1979, ces juvéniles n'ont pas été mesurés ; ils sont représentés par une seule classe de taille $<15 \mathrm{~mm}$. D'autre part, les crevet tes juvéniles présentes dans nos échantillons ont une longueur supérieure ou égale à $10 \mathrm{~mm}$.

Comme le montre la figure 2, les mâles d'Atyephyra desmaresti ont toujours une taille (LT) inférieure à celle de l'ensemble des femelles. Ainsi, au cours de leur croissance, les populations des 2 sexes ont tendance à se séparer, le pic de droite étant toujours représenté par les femelles. Les mâles atteignent une taille maximale de $29 \mathrm{~mm}$ et les classes comprises entre $30 \mathrm{et} 36 \mathrm{~mm}$ sont uniquement constituées de femelles.

C'est en octobre 1977, 1978 et fin septembre 1979 que les juvéniles apparaissent en plus grand nombre dans nos échantillons. C'est également durant ces mêmes mois que les classes de taille $15-20 \mathrm{~mm}$ sont les mieux repésentées, correspondant au recrutement d'une nouvelle génération. A cette période, 2 générations sont présentes dans l'échantillon. Pendant l'hiver, l'abondance des mâles et des femelles de grande taille, correspondant à la génération parentale, diminue. L'année suivante, seule subsiste la nouvelle cohorte dont nous pouvons suivre l'évolution.
La figure 3 illustre la croissance en 1977-1978 des générations apparues pendant ces 2 années. Les classes de taille des différentes cohortes ainsi que leurs longueurs moyennes sont reprises dans le tableau IV. Il faut noter qu'en octobre et en novembre 1978 , la présence simultanée de 2 pics chez les mâles et chez les femelles (fig. 2) correspondant à 2 générations nous incite à suivre leur croissance séparément ; toutefois, vu le léger recouvrement de ces 2 pics, les limites de tailles des différentes cohortes restent imprécises et les résultats obtenus sont plus sujets à caution.

Les mâles et les femelles possèdent le même patron de croissance. En effet, les crevettes nées en 1977 grandissent en automne, ensuite, nous observons un ralentissement (\&) de la croissance pendant l'hiver. Au mois de mars 1978, les femelles et les mâles atteignent respectivement une taille de 25,2 $\mathrm{mm}$ et de $21,2 \mathrm{~mm}$. La croissance reprend au printemps: de mars à juin, l'accroissement moyen des femelles est de $2,1 \mathrm{~mm}$, celui des mâles de $0,9 \mathrm{~mm}$.

Les crevettes cessent ensuite de grandir pendant les mois de juin, juillet et même aoùt dans le cas des femelles. C'est de septembre à octobre que l'accroissement moyen est le plus élevé, à savoir $3,5 \mathrm{~mm}$ dans le cas des femelles et $2,3 \mathrm{~mm}$ dans le cas des mâles. 


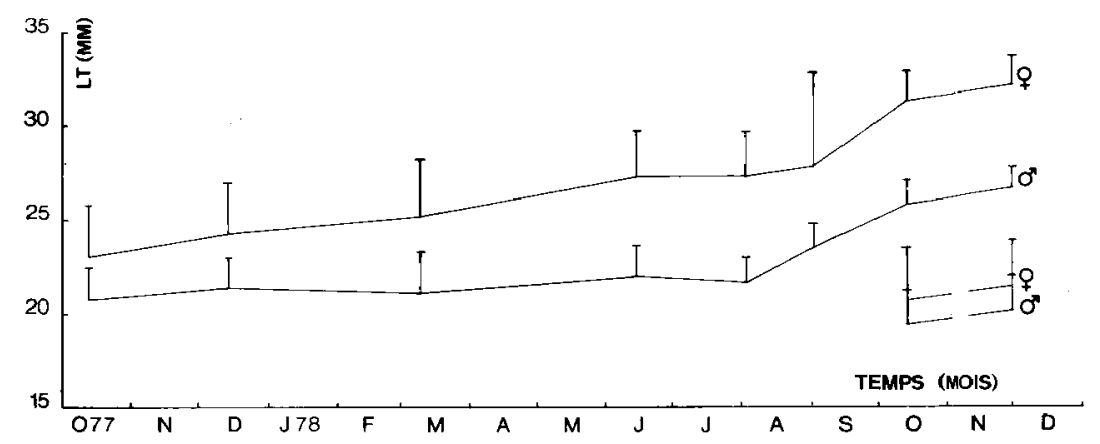

Fig. 3. Croissance en longueur $\left(\mathrm{L}_{\mathrm{T}}: \mathrm{mm}\right)$ des différentes générations de crevettes Atyephyra desmaresti( $\rho$ et $\sigma$ ) en 1977 (- - et 1978 (-) dans la Meuse (moyenne et écart-type).

La vitesse de croissance diminue ensuite au cours du mois d'octobre et de novembre. A la fin de ce mois, les femelles et les mâles atteignent respectivement une taille moyenne maximale de $32,2 \mathrm{~mm}$ et de $26,7 \mathrm{~mm}$. Par la suite, ces individus ne sont plus retrouvés dans les échantillons. Par ailleurs, d'octobre à novembre 1978, nous pouvons également suivre la croissance de la nouvelle génération née durant cette année. Fin novembre, la longueur moyenne des femelles et des mâles de cette cohorte est respectivement de $21,5 \mathrm{~mm}$ et de $20,2 \mathrm{~mm}$. Ces moyennes sont inférieures à celles observées en 1977 au même moment.

De plus, les femelles ont toujours des tailles plus élevées que celles des mâles.

\section{4. - Croissance en poids}

La figure 4 retrace l'évolution du poids moyen des mâles et des femelles capturés en 1977-1978. Ces valeurs sont reprises dans le tableau IV. Chez les individus nés en 1977, le poids varie peu d'octobre a fin mai ; la moyenne chez les femelles se situe aux environs de $100 \mathrm{mg}$ pendant cette période tandis que celle des mâles se stabilise à $60-70 \mathrm{mg}$. Par la suite, la situation évolue peu chez ces derniers et, bien que la croissance en longueur ait repris de façon spectaculaire au cours du mois d'août (fig. 3 ), le poids moyen des mâles n'atteint quand à lui que $75 \mathrm{mg}$. Par contre, dans les prélèvements de mi-octobre, nous observons une hausse sensible du poids moyen des o qui passe alors de 75 à $116 \mathrm{mg}$. Cet te période de croissance se prolonge, mais dans une moindre mesure, pendant le mois d'octobre et vraisemblablement en novembre (poids moyen maximal $=137$ $\mathrm{mg}$ ).

Dans le cas des femelles, il faut attendre le mois de juin, moment d'apparition des œufs chez la plupart des fernelles $(78 \%$ ), pour enregistrer une hausse brutale du poids moyen. En l'espace d'un mois, celui-ci passe en effet de 100 à $170 \mathrm{mg}$, ce qui représente un gain de poids de $70 \%$. Ensuite, de juin à fin aout, le poids des femelles semble se stabiliser aux environs de $160 \mathrm{mg}$. C'est de septembre à mi-octobre, comme dans le cas des mâles, que nous enregistrons un gain de poids très élevé (72 mg).

Fin novembre, les femelles d'Atyephyra desmaresti atteignent en moyenne un poids maximal de $250 \mathrm{mg}$. 


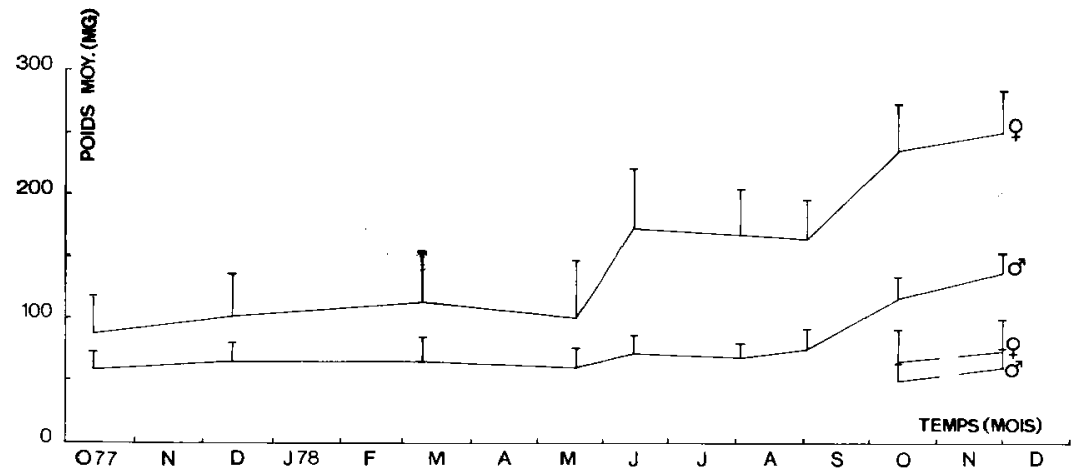

Fig. 4. Evolution du poids moyen des o et des 9 d'Atyephyra desmaresti dans la Meuse, en 1977 (—) et 1978 (--) (moyenne et ćcart-type).

Tableau IV. Longueurs totales moyennes, poids moyens et écarts-types (s) des différentes générations d'Atyephyra desmaresti, capturées en 1977, 1978 dans la Meuse.

\begin{tabular}{|c|c|c|c|c|c|c|c|}
\hline Date & Sexe & $\begin{array}{l}\text { Classe de } \\
\text { taille (mm) }\end{array}$ & $\mathbf{N}$ & $\mathrm{L}_{\mathrm{T}}(\mathrm{mm})$ & $\mathrm{s}$ & Poids (g) & $\mathrm{s}$ \\
\hline \multirow[t]{2}{*}{$12 / 10 / 77$} & $\sigma$ & $15-24$ & 150 & 20,7 & 1,7 & 58,2 & 14,0 \\
\hline & $\%$ & 15.29 & 141 & 23,0 & 2,8 & 87.5 & 31,0 \\
\hline \multirow{2}{*}{$13 / 12 / 77$} & $\sigma$ & $17-27$ & 122 & 21,4 & 1,6 & 65.4 & 14,8 \\
\hline & 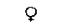 & $17-30$ & 150 & 24,3 & 2,7 & 101,8 & 34,3 \\
\hline \multirow{2}{*}{$09 / 03 / 78$} & $a$ & $15-27$ & 153 & 21,1 & 2,2 & 65,2 & 19,9 \\
\hline & $q$ & $16-32$ & 150 & 25,2 & 3,0 & 113,4 & 39,8 \\
\hline \multirow[t]{2}{*}{$19 / 05 / 78$} & $\sigma$ & 14.25 & 146 & - & : & 60,3 & 16,1 \\
\hline & \& & 14.29 & 150 & . & - & 100,8 & 46,3 \\
\hline \multirow{2}{*}{$14 / 06 / 78$} & $\sigma$ & $17-25$ & 145 & 22,0 & 1,6 & 71,7 & 14,7 \\
\hline & $\phi$ & $18-32$ & 150 & 27,3 & 2,4 & 172,4 & 48,6 \\
\hline \multirow[t]{2}{*}{$02 / 08 / 78$} & or & $19-24$ & 53 & 21,6 & 1,4 & 68,3 & 11,4 \\
\hline & \& & $19-31$ & 150 & 27,3 & 2,4 & 167,1 & 37,1 \\
\hline \multirow[t]{2}{*}{$01 / 09 / 78$} & $\sigma$ & $20-27$ & 150 & 23,5 & 1,3 & 75,2 & 16,6 \\
\hline & \& & $23-32$ & 150 & 27,8 & 5,0 & 163,7 & 31,9 \\
\hline \multirow[t]{4}{*}{$13 / 10 / 78$} & $\sigma$ & 17.23 & 93 & 19,4 & 1,8 & 49,0 & 14,2 \\
\hline & & $23-28$ & 57 & 25,8 & 1,3 & 116,2 & 18,0 \\
\hline & 9 & 17.27 & 69 & 20,7 & 2,8 & 64.8 & 26,2 \\
\hline & & 27.35 & 81 & 31,3 & 1,6 & 235,0 & 37,2 \\
\hline \multirow[t]{4}{*}{$30 / 11 / 78$} & $\sigma$ & $16-24$ & 127 & 20,2 & 1,8 & 60,8 & 15,2 \\
\hline & & $25-28$ & 23 & 26,7 & 1,1 & 137,0 & 15,9 \\
\hline & 8 & $17-28$ & 122 & 21,5 & 2,4 & 74,1 & 25,6 \\
\hline & & $28-35$ & 28 & 32,2 & 1,5 & 250,0 & 33,8 \\
\hline
\end{tabular}


Tableau V. Pourcentage d'apparition des ceufs sur la crevette Arephyra desmaresi dans la Meuse en 1978 et 79 .

\begin{tabular}{ccc}
\hline Date de capture & NQ & Qovigères (\%) \\
\hline 09.03 .78 & 150 & 0 \\
19.05 .78 & 150 & 2 \\
14.06 .78 & 150 & 81.3 \\
02.08 .78 & 150 & 79.4 \\
31.08 .78 & 150 & 2.7 \\
28.06 .79 & 150 & 95.3 \\
21.08 .79 & 111 & 31.5 \\
26.09 .70 & 150 & 0 \\
\hline
\end{tabular}

\section{5. - Reproduction}

\section{1. - Periode de reproduction}

En 1978, dès le mois de mai, $2 \%$ des femelles portent des oufs (Tableau V). Le pourcentage des femel-

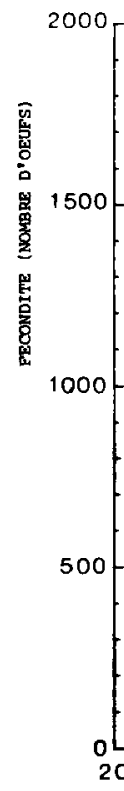

les ovigères augmente par la suite et reste élevé jusque début aoút. Ensuite, ces pourcentages chutent, et fin aoút, seuls $2,7 \%$ de femelles portent encore des cufs.

En 1979. le moment d'apparition des cufs n'a pu ètre précisé, puisque le 28 juin, les premières femel. les capturées cette année-là sont déjà ovigères. Néanmoins, comme en 1978, c'est au mois de juin que le pourcentage de ces femelles est le plus élevé. Fin septembre, plus aucune femelle ne porte des cufs. La taille minimale observee des femelles matures est de $22 \mathrm{~mm}$.

La période de reproduction s'étend donc sur une période de 4 mois : mai-juin-juillet et aoút.

\subsection{2. - Fécondité}

Chez les crustacés décapodes, la fécondité peut ètre estimée par le nombre d'œufs que la femelle porte at tachés aux pléopodes (Mason 1975, in Mason 1977): 
La relation entre la fécondité et la taille des crevettes a été établie en comptant les ceufs de 69 femelles ovigères, prélevées d'un échantillon de juin 1979. Le nombre d'cufs varie entre 347 et 1499 , pour des crevettes dont la taille oscille entre 24 et $35 \mathrm{~mm}$. La fécondité de la caridine varje linéairement avec la taille (fig. 5).

La relation peut être exprimée par l'équation suivante :

$$
\begin{array}{ll}
\mathbf{F}=-1348,9+77,36 \mathrm{~L} & \mathbf{F}: \text { fécondité } \\
& \mathbf{L}: \text { longueur totale en mm }
\end{array}
$$

Le coefficient de corrélation $r$ est de 0,83 .

\section{6. - Régime alimentaire}

\subsubsection{Composition du régime alimentaire}

Un total de 166 contenus digestifs a été examiné au cours de l'année 1979-1980. L'analyse du régime alimentaire (Tableau VI) par la méthode d'occurrence révèle une préférence marquée pour les détritus $(100 \%$ Oc.) et les diatomées $(71 \%)$. Les chlorophytes $(18,7 \%)$, les cyanophytes $(6,0 \%)$ et les protozoaires $(5,4 \%)$ sont effectivement moins souvent consommés. La méthode numérique confirme l'allure générale de ces préférences alimentaires en accentuant l'importance des détritus $(98,3 \%)$ et en soulignant parmi les algues, une consommation plus

Tableau VI. Composition du régime alimentaire d'Atyephyra desmaresti dans la Meuse. à Amay.

\begin{tabular}{lcc}
\hline Taxons & Occurrence & \% Numérique \\
\hline Détritus & 100 & 98.3 \\
Diatomées & $\mathbf{7 1}$ & $\mathbf{1 . 4}$ \\
- Cyclotella meneghiniana & 3.6 & 0.02 \\
- Melosira varians & 21.0 & 0.23 \\
- Diatoma vulgare & 4.8 & 0.04 \\
- Fragillaria spp. & 2.4 & 0.14 \\
- Achnanthes lanceolata & 8.4 & 0.09 \\
- Cocconeis sp. & 12.0 & 0.08 \\
- Synedra & & 0.23 \\
- Navicula spp. & 24.0 & 0.00 \\
- Amphipleura sp. & 1.2 & 0.09 \\
- Rhoicosphenia curvata & 12.0 & 0.08 \\
- Gomphonema spp. & 11.4 & 0.03 \\
- Cymbella ventricosa & 5.4 & 0.01 \\
- Amphora ovalis & 2.4 & 0.01 \\
- Nitzchia & & 0.00 \\
- Surirella spp. & 2.4 & 0.14 \\
Chrosigma sp. & 0.6 & 0.00 \\
- Coelastrum sp. & 18.7 & 0.01 \\
- Pediatrum spp. & 1.2 & 0.01 \\
- Cosmarium spp. & 1.8 & 0.07 \\
- Scenedesmus spp. & 1.8 & 0.04 \\
- Crucigenia spp. & 9.0 & 0.06 \\
Cyanophyles & 5.4 & 0.05 \\
- Ocillatoria spp. & 6.0 & 0.01 \\
- Chamaesiphon incrustans & 4.2 & $\mathbf{0 . 0 3}$ \\
Protozoaires tentaculifères & 1.8 & 0.03 \\
- Acineta sp. & $\mathbf{5 . 4 2}$ & 166 \\
Nombre d'estomacs & 5.42 & \\
\hline & 160 & \\
\hline
\end{tabular}


importante de diatomées $(1,4 \%)$ par rapport aux chlorophytes $(0,14 \%)$ et aux cyanophytes $(0,016 \%)$.

Parmi les 16 genres de diatomées présentes, Nitzchia spp., Navicula spp. et Melosira varians sont le plus consommées. Par contre, d'autres espèces telles Gyrosigma sp. et Amphipleura sp. n'apparaissent que très rarement dans les contenus stomacaux. Les chlorophytes sont représentees seulement par des algues microscopiques non filamenteuses: Scenedesmus est la plus fréquente et la plus abondante. Crucigenia, Pediatrum, Cosmarium et Coelastrum sont plus rarement consommées. les cyanophytes ne représentent qu'un très faible apport dans le régime alimentaire d'Atyephyra. Oscillatoria spp. et Chamaesiphon incrustans sont les seules espèces obser- vées dans les estomacs. Quand aux détritus, ils consistent principalement en du matériel très petit d'origine végétale en état avancé de décomposition.

Ce sont également des restes d'algues vertes filamenteuses indéterminables et des fragments de val. ves de diatomées. Les détritus d'origine animale sont représentés par des spicules d'éponge, des restes de cuticule et des soies de microinvertébrés.

4.6.2. Variations du régime alimentaire au cours des saisons

De l'observation du tableau VII, il ressort que les variations dans la composition de la nourriture sont

Tableau VII. Composition du régime alimentaire (\% numérique) d'Atyephyra desmaresti de la Meuse (Ombret) à différentes périodes de l'année.

\begin{tabular}{|c|c|c|c|c|c|}
\hline Taxons & $02 / 08 / 79$ & $21 / 08 / 74$ & $25 / 10 / 79$ & $31 / 01 / 80$ & $22 / 04 / 80$ \\
\hline Détritus & 99.6 & 98.2 & 98.7 & 100 & 96.7 \\
\hline Diatomées & 0.27 & 1.3 & 1.1 & 0.00 & 3.2 \\
\hline - Cyclotella meneghiniana & & 0.01 & 0.03 & & 0.03 \\
\hline - Melosira varians & 0.13 & 0.23 & 0.30 & & 0.19 \\
\hline - Diatoma vulgare. & 0.01 & 0.01 & 0.02 & & 0.13 \\
\hline - Fragillaria spp. & 0.01 & 0.07 & 0.13 & & 0.32 \\
\hline - Achnanthes lanceolata & . & . & 0.03 & & 0.38 \\
\hline - Cocconeis sp. & 0.01 & 0.19 & 0.05 & & 0.07 \\
\hline - Synedra spp. & 0.01 & 0.01 & 0.05 & & 0.03 \\
\hline - Navicula spp. & - & 0.17 & 0.06 & & 0.84 \\
\hline - Amphiplesira sp. & - & 0.01 & 0.01 & & \\
\hline - Rhoicosphenia curvala & 0.03 & 0.14 & 0.04 & & 0.19 \\
\hline - Gomphonema spp. & 0.01 & 0.08 & 0.05 & & 0.19 \\
\hline - Cymbella ventricosa & - & 0.01 & 0.01 & & 0.13 \\
\hline - Amphora ovalis & - & 0.03 & 0.01 & & - \\
\hline - Nitzchia spp. & 0.01 & 0.19 & 0.26 & & 0.65 \\
\hline - Strivella spp. & - & 0.01 & 0.03 & & - \\
\hline - Gyrosigma sp. & - & 0.1 & - & & . \\
\hline Chlorophytes & 0.07 & 0.21 & 0.20 & 0.00 & 0.01 \\
\hline - Coclastrum sp. & . & - & 0.02 & & . \\
\hline - Pecliatrum spp. & - & 0.03 & 0.01 & & - \\
\hline - Cosmarizm spp. & - & 0.01 & 0.02 & & - \\
\hline - Scenedesmus spp. & 0.05 & 0.12 & 0.06 & & 0.01 \\
\hline - Crucigemia spp. & 0.01 & 0.01 & 0.08 & & - \\
\hline Cyanophytes & 0.00 & 0.17 & 0.05 & 0.00 & 0.00 \\
\hline $\begin{array}{l}\text { - Oscillatoria spp. } \\
\text { - Chamaesiphor incrustans }\end{array}$ & & & $\begin{array}{l}0.02 \\
0.03\end{array}$ & & \\
\hline Protowaires tentaculiferes & 0.01 & 0.07 & 0.02 & 0.00 & 0.01 \\
\hline - Acineta sp. & 0.01 & 0.07 & 0.02 & 0.00 & 0.01 \\
\hline Nombre d'estomacs & 25 & 50 & 61 & 21 & 30 \\
\hline
\end{tabular}


très faibles. Toutefois, la simple observation des estomacs indique que le volume de nourriture est plus faible en hiver. Mais quelle que soit la saison, on rencontre toujours une prédominance de détritus ( $100 \%$ Oc. et $96.7 \%$ à $\mathrm{N})$.

Les algues sont, cependant, absentes dans les estomacs de crevettes capturées en hiver, alors qu'au printemps, les diatomées font leur apparition.

En été, parmi les algues, ce sont les chlorophytes et les diatomées qui sont les plus consommées.

\section{5. - Discussion}

\section{1. - Abondance de la crevette}

Les prélèvements effectués depuis octobre 1977 jusque octobre 1980 ont mis en évidence une diminution de l'abondance d'Atyephyra desmaresti sur les berges de la Meuse, en 1979 et surtout en 1980. La rarefaction de cette espèce ne se limite pas au seul secteur étudié. En effet, des observations réalisés sur les rives au cours du chômage technique de la Meuse en amont de Namur, montrent également une nelle diminution de l'abondance de cet atyidè en 1980 par rapport à 1977.

Parmi la population vivant dans le fleuve à la station d'Amay, les femelles sont toujours plus abondantes que les mâles, et cette différence s'accentue au cours de la période de reproduction.

\section{2. - Cycle vital et croissance}

Atvephyra desmaresti est une espece univoltine. Au cours de l'été apparaissent les jeunes qui grandissent jusqu'à la fin de l'automne. Puis, its passent par une période $d$ 'hibernation qui se traduit par un arrêt de la croissance. Cette croissance des jeunes pendant les mois d'aoút, septembre et octobre, de même que le ralentissement voire l'arrêt de la croissance pendant l'hiver ont également été constatés dans un canal à Amsterdam (Vorstman 1955) et dans la Moselle (Packa-Tchissambou 1979). Descouturelle (1976 in Packa-Tchissambou 1979) a observé un blocage des mues pendant la période hivernale des crevettes vivant dans le canal de la Marne au Rhin. Ce blocage est responsable de l'arrêt de la croissance. En laboratoire, ce même auteur a montré que des basses températures (inférieures à $5^{\circ} \mathrm{C}$ ) provoquaient un allongement du cycle d'intermue (Descouturelle 1976). Au printemps, le taux de croissance en longueur reste très faible, ensuite il s'annule pendant la période de reproduction. Après cette periode, les individus continuent à grandir : c'est de septembre à octobre que la vitesse de croissance est la plus élevée. Ils deviennent ensuite sénescents et meurent en automne et en hiver. Dans des eaux réchauffées du site de la Maxe, Packa-Tchissambou (1979) a également mis en évidence 3 périodes de croissance : une croissance rapide au cours des mois précédents l'hiver (de juillet à novembre), un ralentissement de la croissance en hiver, une reprise de la croissance en février, qui reste faible jusqu'à la fín du cycle de reproduction (juin). Toutefois, dans ces eaux réchauffées, la plupart des crevettes meurent après la reproduction. Dans la Meuse, la durée de vie de cet atyidé semble légèrement plus longue et elle se situerait entre un an et un an et demi. Aux Pays-Bas, Vorstman (1955) a montré que des conditions défavorables de température et de luminosité (printemps froid et sombre) pouvaient induire un allongement du cycle vital : les crevettes devenaient matures seulement après 2 ans. Les longueurs totales maximales atteintes en 1977-1980 par les femelles et les máles en fin de période de croissance en Meuse sont respectivement de 36 et $29 \mathrm{~mm}$. Des tailles maximales de $34 \mathrm{~mm}$ et de $35 \mathrm{~mm}$ ont été mesurées pour des crevettes femelles vivant respectivement dans un canal à Amsterdam (Vorstman 1955), et dans la Moselle (Packa-Tchissambou 1979).

Ouant à la croissance en poids, après un arrêt hivernal de novembre 1977 à la fin mars 1978 , celleci reprend en été. Le gain en poids est plus marqué chez les femelles du fait de l'apparition des ceufs. Mais si cette apparition accentue l'importance de la prise de poids, il existe en plus une croissance de l'individu lui-même. En effet, si nous considérons uniquement les femelles sans oufs, leur poids moyen se situe à environ $117 \mathrm{mg}$ à la mi-juin et à $138 \mathrm{mg}$ début août. A chaque période de l'année, les femelles sont plus grosses que les máles. Les poids moyens maxima atteints en 1978 par les femelles et les máles dans la Meuse en fin de période de crois. sance sont respectivement de 250 et de $137 \mathrm{mg}$. Les longueurs moyennes correspondantes sont de 32 et $27 \mathrm{~mm}$. 


\section{3. - Reproduction}

Dans la Meuse, la période de reproduction s'étend sur 4 mois : mai, juin, juillet et aout. Elle débute lors. que la température de l'eau se rapproche de $15^{\circ} \mathrm{C}$. En juin, et probablement en juillet, la reproduction apparait très active. Descouturelle (in Nourisson \& al. 1978) observe la même période de reproduction d'Atyephyra desmaresti vivant dans le canal reliant la Marne au Rhin. Une durée de 5 mois (mai à septembre) est observèe par Ghalano (1979) dans la rivière Dauso, au Portugal.

Dans un canal d'Amsterdam, cet atyidé se reproduit pendant les mois de mai, juin et juillet (VorsIamn 1955).

Dans la Meuse, il semble que la taille minimale des fermelles à maturité soit de $22 \mathrm{~mm}$; ceci a été observé également par Gauthier (1924) et Nouvel (1940).

La relation fécondité-ıaille (en données arithmétiques) est généralement de type linéaire chez les décapodes. Brown \& Bowler (1977) la signalent pour Austropotamobius pallipes et Abrahamsson (1972) pour Astacus astacus (in Brown \& Bowler 1977). Chavez-Alarcon et al. (1976) trouvent une relation de ce type chez Macrobrachium carcinus L. En ce qui concerne les Atyides, Williams (1977) a signale chez. Puratya austramiensis une relation linéaire entre le nombre d'ceufs et la taille de la femelle.

Dans la Meuse, la fécondité varie entre 347 et 1499 pour des crevettes dont la taille oscille entre 24 et $35 \mathrm{~mm}$. Une fécondité comparable est trouvéc par Gauthier (1924 in Vorstman 1955).

\section{4. - Régime alimentaire}

Le régime alimentaire d'Atyephyra desmaresti con. siste principalement en détritus. Ce régime détritivore est d'ailleurs caractéristique des Atyides. (Fryer 1977, Bouvier 1925, Darnelle 1956). Selon ces deux derniers auteurs, tous les Atyidés (sauf Xiphocaris) possèdent une touffe de soies à l'extrémitć de's chélipedes dont la fonction est de collecter des detritus composés de microparticules. L'importance nutritionnelle des détritus d'origine végétale résulte de l'abondance de cellulose et d'amidon, 2 sources d'hydrates de carbone et d'énergie, qui peuvent étre utilisées moyennant la présence de bactéries amylolytiques et hydrolytiques dans le substrat (Darnell 1964). Les variations saisonnières du régime alimentaire sont en relation avec la disponibilité de ces aljments dans le milieu.

Toutefois, comme la crevette se nourrit à l'aide de chélipèdes munis de fortes touffes de soies, les organismes animaux et végétaux trouvés dans l'estomac ont pu être ingérés en même temps que les particules en décomposition et ne révèlent pas forcément les préférences alimentaires. Dans les fécès, on trouve des algues non digérées.

En conclusion, Aryephyra desmaresti est un animal fondamentalement détritivore, qui trouve sa nourriture en brossant les algues vertes filamenteuses (Cladophora) présentes sur les cailloux du fleuve.

\section{Remerciements}

Nous remercions Y. Mine pour sa collaboration technique.

\section{Travaux cltés}

Adam (W.) \& Leluup (A.E.). 1940. - La distribution en Belgique de Leticochloridim paradoxm et de Atrephyra desmaresti Millet. Bull. Mus. Hist. Nat. Belg., $16(21)$ : 1.8.

Bourrelly (P.). 1968. - Les algues d'eau donce. Tome II : Les algues jaunes et brunes. Ed. Boubee \& Cie, Paris.

Bourrelly (P.). 1970. - Les algates d'eat donce. Tome 11 : Les algues blentes et roukes. Ed. Boubere \& Cie, Paris

Bourrelly (P.) 1972. - Les algates d'at dotce. Tome II : Les algues reries. Ed. Buuber \& Cie, Paris

Brown (D.S.) \& Bowler (K.). 1977. - A population study of bri(ish freshwater cravfish Austropotamobits pallipes (Lerebaullet). Freshluater crayfish. Papers from the Third International Simposium on Freshwater cravfish, Finland, $1966: 3: 33-49$

Chave\%-Alarcon (Z.) \& al., 1976 - Iniruduction al conocimiento de la biologica del langostino (Macrobachitun carcinus L.) en cl estade de Veracruz. Men. Simp. Biel. Dinam. Probl. de Cama rom. Guavmas. Somestel: $13-23$

Dasnell (R.M.). 1956. - Analysis of a population of the tropical freshwaler shrimps, Atyes scahra (Leach). Am. Midl. Nat., 55 : 131.138 
Darnell (R.M.). 1964 . - Organic detritus in relation to secondary production in aquatic communities. Verh. Inter., Verein. Lim. mol. XV : $462-470$

Declercq-Versele (J.), Kirchmann (R.) \& al., 1982. - L'impact des rejets de la centrale nucléaire de Tihange (Belgique) sur l'écosystème Meuse : cinq années d'êtude in situ et d'approche expérimentale (1976-1980). BLG 555: $54 \mathrm{p}$

Descouturelle (G.) 1974 - Différenciation des caractères sexuels femelles chez la crevette d'eau douce Atyephyra desmaresti Millet. (Crustacea, Décapode. Natantia). Cr. Séanc-Soc. Biol. de Nancy, $169(6)$ : 1412-1416

Descouturelle (G.). 1976. - Influence de la température et de la sexualité sur la durée des stades d'intermue chez la crevefte d'eau douce Atyephyra desmaresti Miliet. Vie Miliet, XXVI (2) Sér, C. 149-172.

Fryer (G.) 1977. - Studies on the functionnal morphology and ecology of the atyid prawns of Dominica. Phil. Trans. R. Soc; (B), $177: 57.129$.

Gauthier (H.). 1925. - Recherches sur le développement larvaire d'Atyephyra desmaresti (Millet 1832) (Décp. Nat., Car, Atyides) : Bull., Soc. Hist. Nat. Afrique du Nord. 15 : 335.376.

Ghalano (H.) 1979. - Seasorial changes in Atyephyra desmaresti Millet (Decapoda Natantia). Extracto des fases. $1^{\circ}$ a $4^{\circ}$ do volume LXI des Anais da Facaldade de Ciencias do Porto. $26 \mathrm{p}$.

Lestage (S.A.). 1931. - Note de Limnologie. I. L'habitat en Belgique de Atyephyra desmaresti. Ann. Soc. Roy. Zool. Belgigute, 12 : 53.58.

Mason (J.). 1977, - Reproduction efficiency of Pascifastacus Teniusculus (Dana) in culture. Freshwater Crayfish, 3 : 101-117.
Minshall (G.) 1967. - Role of allochtonous detritus in the trophic structure of a woodland springbrook communily. Ecology, 48: 139-149.

Nourrison (M.)\& Tchissambou (B.). 1978. - Influence de la température sur l'evolution d'une population de Caridines (Atyephyra desmaresti Millet) du site de la Maxe : résultats préliminaires. Cah. Lab. Montereati. $7: 47.52$.

Nowel (L.) 1940. - Observations sur la biologie de l'Atyephyra desmaresti Bull. Soc. Hist. Nat., Toulouse, 75 : 243-252.

Packa-Tchissambou (B.). 1979. - Etude " in situ des effets de la temperature sur la biologie de la crevetle d'eau douce Atyephyra desmaresti Millel : cas particulier du bassir de rejet de la centrale thermique de la Maxe. Thèse 3e cycle, Univer. sité de Me1z, $91 \mathrm{p}$.

Perrier (R.). 1971. - La faune de la France. Tome I A : Coelente. rés-Spongiaires. Echinodermes-Prolozoaires. Ed. Delagrave, Paris : $92-94$

Prescott (G.W.). 1979. - The freshwater algae. Pictural key Nature series. WMC Brown Company publishers Iova.

Redeke (H.). 1936. - La crevelte d'eau douce, Atyephyra desmaresti (Mill.) dars les Pays-bas. Mem. Mus. Hist. Nat. Belg., 2 (3): 227-231.

Vorstman (A.G.). 1955. - Investigation on the life cycle of Alyephyra desmaresti (Millet). Verh. Int. Verein., Limnol 12 : 469.477 .

Wiltiams (D.W.). 1977. - Some aspects of the Ecology of Paratya atstraliensis (Crustacea: Decapoda : Atyidae), Alist. J. Mar. Freshwater. Res., 28 : 403-415. 